\title{
Dyspnoea, anorexia and weight loss in a 74 year old man
}

\author{
L.G. Heaney, I.C. Gleadhill
}

\section{Case history}

A 74 year old male, whilst on holiday, was admitted with a history of increasing dyspnoea on exertion and associated left-sided pleuritic chest pain. He reported that for 2 months he had experienced mild discomfort in the lumbar area, and that 5 weeks previously he had complained of upper abdominal pain and attended an emergency department. Clinical examination and electrocardiogram (ECG) were normal, and a diagnosis of oesophageal reflux was made. The patient was commenced on an antacid, with some initial improvement. He also had a 2 month history of anorexia and weight loss. He had a $20 \mathrm{yr}$ history of hypertension and was an ex-smoker, having smoked 15 cigarettes $\cdot$ day $^{-1}$ for 20 yrs.

On examination, he was pyrexial $\left(38.5^{\circ} \mathrm{C}\right)$ and anaemic. Cardiovascular examination was unremarkable, but examination of the chest revealed dullness to percussion at the left base, reduced breath sounds, and decreased vocal resonance. A chest radiograph on admission is presented in figure 1. Investigation revealed a haemoglobin level of $99 \mathrm{~g} \cdot \mathrm{L}^{-1}$ with normocytic normochromic indices, leucocyte count $10.0 \times 10^{9}$ cells $\cdot \mathrm{L}^{-1}$, urea 12.5 $\mathrm{mmol} \cdot \mathrm{L}^{-1}$, and creatinine $168 \mu \mathrm{mol} \cdot \mathrm{L}^{-1}$. An initial differential diagnosis of pneumonia, with possible underlying bronchogenic carcinoma or pulmonary embolism, was made. The patient was heparinized and commenced on a broad spectrum penicillin. Initially, his condition improved, with temperature settling and improvement in his dyspnoea and chest pain.

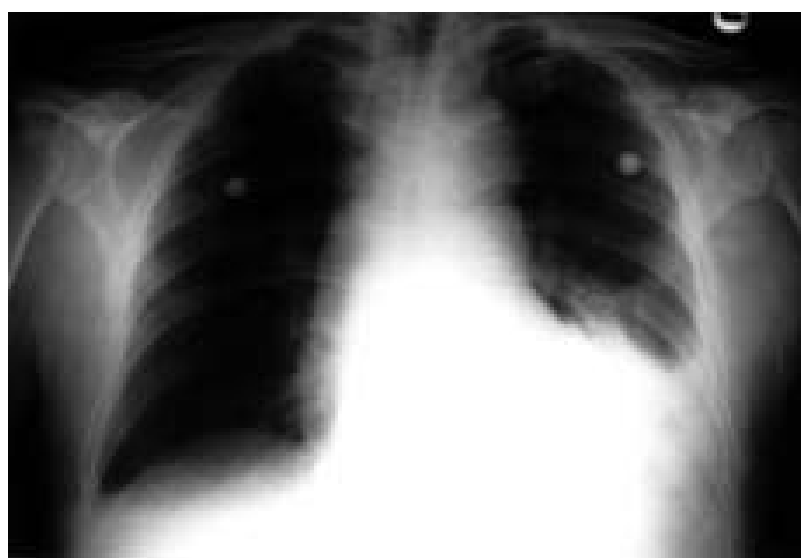

Fig. 1. - Chest radiograph on admission to hospital. Anteroposterior view, with the patient in semi-recumbent position.

Correspondence: L.G. Heaney, Dept of Respiratory Medicine, Level 8, Belfast City Hospital, Lisburn Road, Belfast, Northern Ireland, BT9 $7 \mathrm{AB}$. a)

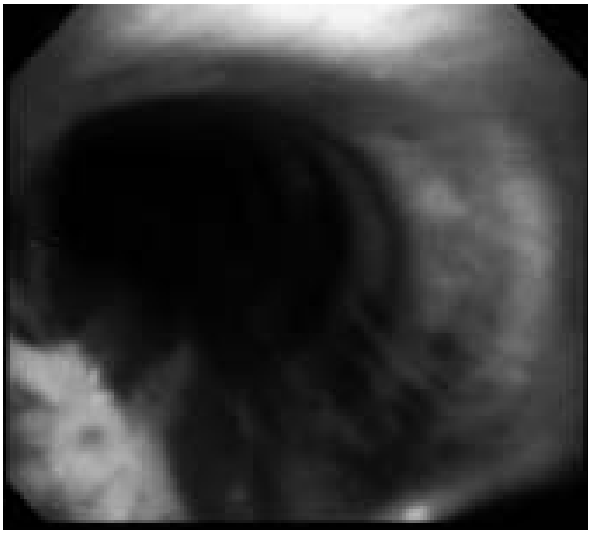

b)

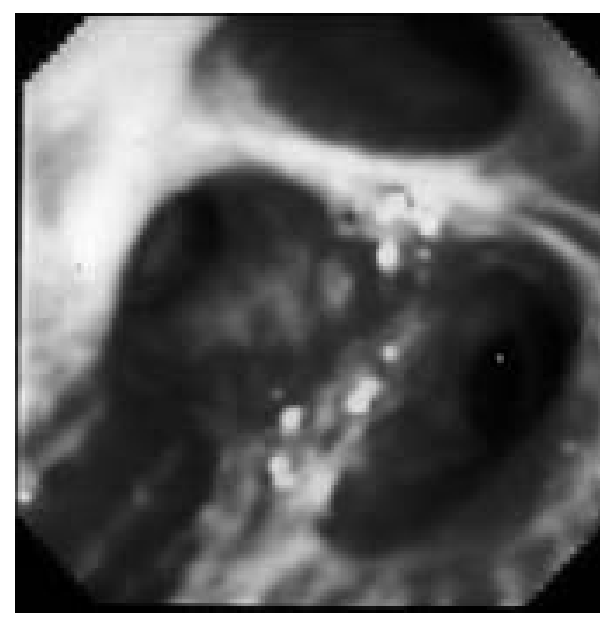

c)

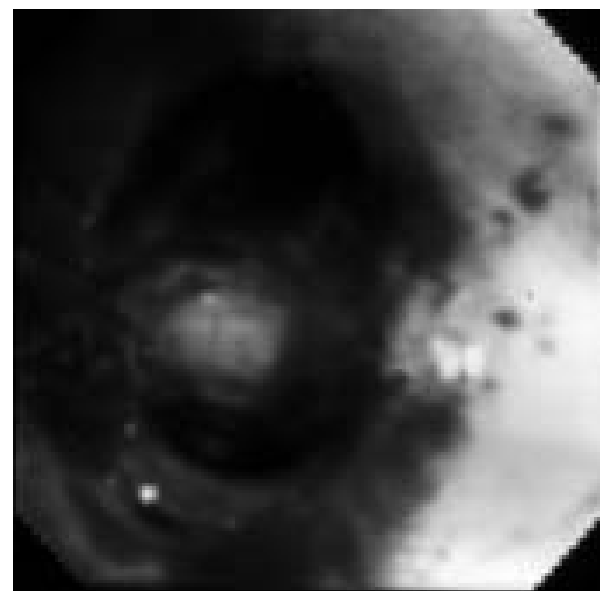

Fig. 2. - Bronchoscopic appearance of: a) left main bronchus; b) right upper lobe bronchus; and c) left upper division. The photographs of each site were taken before passage of the bronchoscope, thus appearances are not due to scope trauma. 
The patient was transferred to his local hospital from his holiday location, where, on review of the history, a diagnosis of left lower pneumonia and parapneumonic effusion was made, with the possibility of an underlying neoplasm. The heparin was discontinued and a pleural aspiration performed. This revealed a bloodstained effusion with a protein level of $42 \mathrm{~g} \cdot \mathrm{L}^{-1}$, but no malignant cells were identified. The patient remained stable with only minor pleuritic chest discomfort and, in view of his smoking history, presumptive lower lobe pneumonia and nondiagnostic pleural aspiration, bronchoscopy was arranged. At bronchoscopy, no focal endobronchial abnormality was identified; however, it was noted that the mucosa was oedematous and had diffuse linear streaks of submucosal blood from the level of the main carina, which became confluent at segmental and subsegmental level (fig. 2). No vessels were identified. In view of this rather strange appearance, an urgent computed tomography (CT) scan of the thorax was performed (fig. 3).

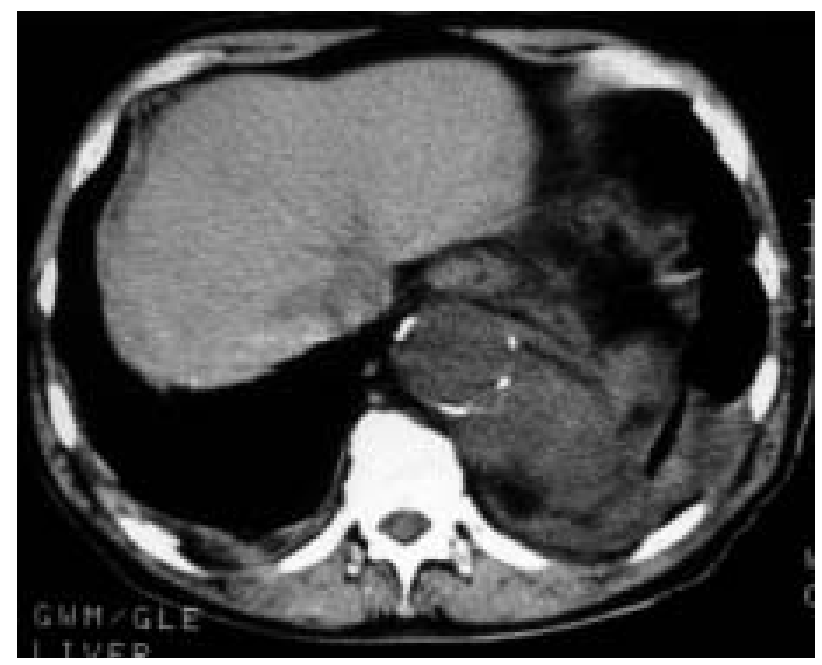

Fig. 3. - Computed tomography (CT) scan of the thorax.

\section{BEFORE TURNING THE PAGE: INTERPRET THE FIGURES AND SUGGEST DIAGNOSIS AND TREATMENT.}




\section{Interpretation of chest radiograph and CT scan}

The chest radiograph (fig. 1) was obtained with the patient in the semi-recumbent position. It shows a left pleural effusion and cardiomegaly, which may in part relate to the anteroposterior view. Analysis of the pleural aspirate demonstrated a bloodstained exudate, with no malignant cell population.

The CT scan (fig. 3) demonstrates a calcified descending thoracic aorta and left pleural effusion. CT of the aorta at the level of the aortic arch was normal. The discomfort in the lumbar area, the upper abdominal pain, the bloodstained pleural effusion, the low haemoglobin value, together with the patient's age and the calcified thoracic aorta all indicate a ruptured aneurysm of the descending thoracic aorta.

\section{Diagnosis: "Ruptured aneurysm of the descending thoracic aorta."}

\section{Treatment and clinical course}

Surgical repair was undertaken and operative findings were ruptured descending aortic thoracic aneurysm, with distal aneurysmal neck behind the diaphragmatic crura but proximal to the coeliac axis. The overlying left lung was plastered to the continued rupture and difficult to dissect free. Graft replacement to the aneurysmal section was performed.

Postoperative recovery was uneventful and the pleural effusion resolved. The patient was fit and well at subsequent review.

\section{Discussion}

This case highlights the difficulty in diagnosing ruptured aneurysms of the descending thoracic aorta. If no mass has been previously recorded on chest radiography and no compressive symptoms are present, then a previous diagnosis of thoracic aortic aneurysm is unlikely to be made. Compression of the large airways may produce wheezing, cough and pneumonitis distal to the obstruction [1]. Erosion into the pulmonary parenchyma may cause haemoptysis. Compression of the oesophagus may lead to dysphagia, and compression of the recurrent laryngeal nerve to hoarseness [1]. The incidence of rupture of thoracic aneurysms is higher than for abdominal disease. BICKERSTAFF et al. [2] reported a series of 72 patients with thoracic aortic aneurysm observed over a period of $30 \mathrm{yrs}$. The descending thoracic aorta was involved in 27 patients. In the overall group, rupture occurred in 53 patients $(74 \%)$. Of these, 37 (51\% of overall group) had no previous diagnosis of aneurysm and in 16 others the mean interval between diagnosis and rupture was 2 yrs. Actuarial 5 yr survival for all patients was $13 \%$, and for patients without dissection $20 \%$. Thus, when the diagnosis of thoracic aneurysm is made, surgical repair is recommended, and only with small aneurysms and significant coronary or cerebral vascular disease is a nonoperative policy of observation with frequent chest radiographs indicated.
If rupture occurs and the subject survives, then the symptoms are chest and back pain, and signs will be determined by the degree of acute blood loss and resultant hypovolaemia. There are often no clinical findings unless the aneurysm extends into the abdomen, when it may not be possible to palpate the proximal neck of the abdominal aneurysm. In the case outlined above, the blood loss was presumably more chronic, and at no time were there symptoms or signs suggestive of massive blood loss. The only indication of bleeding was the presence of a normocytic normochromic anaemia; however, this together with the ongoing chest and back pain are often compatible with an alternative and more likely clinical diagnosis. The presence of bloodstained pleural effusion was also felt to have a more likely aetiology, particularly with the accompanying pyrexia, weight loss and anorexia. Thus, the diagnosis of ruptured thoracic aneurysm may not always be obvious, and may hence be delayed.

The bronchoscopic appearance has not been reported previously, and we feel it is sufficiently striking and unusual to be highly suggestive, if not diagnostic, of ruptured thoracic aneurysm. In view of the appearance, it is perhaps surprising that the subject did not have haemoptysis. The origin of the submucosal blood observed could be due to a number of different mechanisms, namely: 1) mucosal ischaemia; 2) small vessel haemorrhage due to elevated venous pressure; or 3) blood tracking from the ruptured aneurysm to the bronchial mucosa. The bronchial mucosa between the haemorrhagic areas did not appear ischaemic, and the red "ischaemic" tissue appeared too linear and regular for ischaemia to be the sole mechanism. The presence of mucosal oedema could be due to ischaemia or, alternatively, to an elevation of venous pressure due to compression of pulmonary and bronchial veins from the bleeding aneurysm. This could also cause small submucosal vessels to burst causing patchy submucosal haemorrhage. Free mediastinal blood does track into the pleural space but it seems unlikely that blood would track from outside the airway to the mucosal surface.

Whatever the mechanism, if the bronchoscopic appearance outlined above is observed, we recommend review of the history, consideration of ruptured thoracic aneurysm and, if necessary, computed tomographic scan of thorax.

\footnotetext{
Acknowledgement: The authors gratefully acknowledge the support of Allen and Hanburys in reproduction of the colour prints.
}

Keywords: Aneurysm, aorta, bronchoscopy, thorax.

\section{References}

1. Galloway AC, Colvin SB, Spencer FC. Diseases of the great vessels. In: Schwartz SI, Shires GT, Spencer FC, eds. Principles of Surgery. New York, McGraw-Hill, 1994; pp. 903-924.

2. Bickerstaff LK, Pairolero PC, Hollier LH, et al. Thoracic aortic aneurysms: a population-based study. Surgery 1982; 92: 1103-1107. 